\title{
Les grands programmes expérimentaux de sûreté des installations nucléaires
}

\section{Avant-propos}

\author{
par F. COGNÉ \\ Chef du Département de Sûreté Nucléaire \\ CEA/IPSN
}

$\mathbf{L}^{\circ}$

e principe même de recherches en matière de sûreté est souvent mal perçu et le bien-fondé de grands programmes dans ce domaine mis en doute. Autant l'on saisit d'emblée la nécessité d'études directement applicables à des projets ou des réalisations, autant on peut parfois se poser des questions sur l'utilité de certains programmes de sûreté destinés à explorer des accidents que l'on s'évertue à montrer par ailleurs comme hautement improbables. Les uns se demanderont pourquoi faire de telle dépenses pour des études non directement applicables, les autres diront, avec une logique imparable, que toutes ces études de sûreté doivent (ou auraient dû) être un préalable à toute réalisation. En définitive, l'absence d'accidents graves finit par rassurer tout le monde... et c'est Three Mile Island.

Comment garder la mesure dans tout ceci? L'approche choisie en France pour définir nos programmes expérimentaux a toujours été de chercher à étudier les phénomènes physiques fondamentaux qui peuvent intervenir dans les accidents, et non de tenter des expérimentations "représentatives". Ceci suppose d'être à même de réaliser des expériences analytiques, de mettre au point des moyens d'interprétation (codes), et ensuite d'utiliser ces moyens pour les projets ou les calculs d'accidents sur les installations. Cette méthode d'approche est longue, difficile, peu démonstrative pour le public; mais elle permet de développer des connaissances applicables non seulement aux grands accidents, mais aussi à l'ensemble des études des différentes conditions de fonctionnement des installations. e présent numéro de la RGN arrive à point nommé à un moment charnière des grands programmes expérimentaux en France et même à l'étranger. En effet, l'année 1982 aura été marquée d'un certain nombre d'événements, de résultats ou de décisions que je voudrais résumer car ils sont comme une introduction aux articles présentés.

Dans le domaine des réacteurs à eau d'abord, le programme expérimental thermohydraulique analytique arrive à son aboutissement avec les débuts de mise en service du code CATHARE et le CEA, EDF et Framatome ont décidé d'engager la réalisation d'une "boucle système " capable de représenter l'ensemble des phénomènes physiques survenant lors des différents types d'accidents de dépressurisation envisageables. Par ailleurs, s'appuyant sur le code CATHARE, la réalisation de simulateurs de conduite comportant la physique réelle des accidents a été mise à l'étude. De son côté, le réacteur PHEBUS à Cadarache a réalisé le 8 juillet un accident complet de dépressurisation sur une grappe de vingt-cinq crayons combustibles; cet essai, le dernier de la première phase du programme, conclut toute une série d'essais progressifs qui a duré deux ans. A l'étranger, le programme LOFT décidé après l'accident de TMI se termine, alors que la Communauté Européenne envisage le projet Super-Sara à Ispra.

Pour les réacteurs surgénérateurs, l'année a été marquée par la conférence internationale sur la sûreté de ces réacteurs, organisée par la SFEN, sous le patronage commun de l'ANS et de la SEEN, qui s'est tenue à Lyon du 19 au 22 juillet 1982. Nos grands programmes expérimentaux $y$ ont été largement présentés; il faut souligner en particulier la valeur des essais (six en 1982 dont quatre sur combustible irradié) effectués sur le réacteur CABRI, qui constituent aujourd'hui une référence internationale essentielle pour l'analyse des accidents hypothétiques graves. Deux autres programmes vont franchir des étapes importantes en 1982 : l'installation ESMERALDA (étude des grands feux de sodium), réalisée en commun entre CEA, EDF, NERSA et l'Italie (CNEN), mise en service en septembre; le réacteur SCARABEE, complémentaire de $C A B R I$, destiné d'abord à étudier des accidents de perte de débit dans un assemblage combustible, dont le premier essai doit avoir lieu en fin d'année.

Dans le domaine des études de sûreté-criticité, nécessaires dès que l'on manipule des quantités importantes de matières fissiles dans les usines du cycle du combustible, je signalerai la mise en service à l'automne 1982 de l'installation MARACAS destinée à l'étude de la criticité de grandes masses d'UO faiblement enrichi.

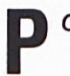

our terminer, il faut souligner à quel point la connaissance en matière de sûreté nucléaire nécessite des échanges ouverts et sans arrière-pensée, bien sûr en raison du coût élevé des études, mais avant tout parce que cette connaissance doit appartenir à tous; il ne serait pas admissible qu'un accident survienne quelque part, alors qu'à côté certains garderaient jalousement leurs connaissances sur les risques encourus. C'est pourquoi l'Institut de Protection et de Sûreté Nucléaire du CEA a toujours tenu à réaliser le maximum possible de ses grands programmes dans un cadre large de coopération internationale. 